\title{
On the Solution of Fractional Maxwell Equations by Sumudu Transform
}

\author{
Adem Kılıçman (Corresponding author) \\ Department of Mathematics and Institute for Mathematical Research \\ Universiti Putra Malaysia, 43400 UPM, Serdang, Selangor, Malaysia \\ Tel: 603-8946-6813 E-mail: akilicman@putra.upm.edu.my \\ V. G. Gupta \\ Department of Mathematics, University of Rajasthan \\ Jaipur-302055, India \\ E-mail: guptavguor@rediffmail.com \\ Bhavna Shrama \\ Department of Mathematics, Shri Balaji Institute of Engineering and Technology \\ Jaipur - 302045, India \\ E-mail: vallabhi_2009@yahoo.com
}

\begin{abstract}
In this paper, we introduce the Maxwell equations of time- fractional order in lossy media. We derive the solution of these equations by using Sumudu transform techniques.
\end{abstract}

Keywords: Sumudu transform, Fractional Maxwell equations, Fractional differential operator, Mittag-Leffler function

\section{Introduction}

In the literature there are numerous integral transforms and widely used in physics, astronomy as well as in engineering. In order to solve the differential equations, the integral transforms were extensively applied and thus there are several works on the theory and application of integral transforms such as the Laplace, Fourier, Mellin and Hankel, to name a few. In the sequence of these transforms, in early 90's Watugala (G. K.Watugala, 1993) introduced a new integral transform, named the Sumudu transform and further applied it to the solution of ordinary differential equation in control engineering problems. For further detail and properties about Sumudu transform (M. A. Asiru, 2001; 2002; 2003 ; F. B. M. Belgacem, 2003; M. E. El-Shandwily, 1988; H. Eltayeb, 2010; V. G. Gupta, 2010) and many others. The Sumudu transform is defined over the set of the functions:

$$
A=\left\{f(t): \exists M, \tau_{1}, \tau_{2}>0,|f(t)|<M e^{t / \tau_{j}}, \text { if } t \in(-1)^{j} \times[0, \infty)\right\}
$$

by the following formula

$$
\bar{f}(u)=S[f(t) ; u]=: \int_{0}^{\infty} f(u t) e^{-t} d t, u \in\left(-\tau_{1}, \tau_{2}\right) .
$$

The existence and the uniqueness was discussed in (A. K1lıçman, 2010), for further properties of Sumudu transform and its derivatives we refer to (M. A. Asiru, 2001). In (M. A. Asiru, 2002), some fundamental further properties of Sumudu transform were also established.

Similarly, this new transform was applied to the one-dimensional neutron transport equation in (A. Kadem, 2005). In fact one can easily show that there is strong relationship between Sumudu and other integral transforms. In particular the relation between Sumudu transform and Laplace transforms was proved in (A. Kılıçman, 2010).

Further in (H. Eltayeb, 2010), the Sumudu transform was extended to the distributions(generalized functions) and some of their properties were also studied in (A. Kılıçman, 2010). Recently Kılıçman et al. applied this transform to solve the system of differential equations, see (A. Kılıçman, 2010).

A very interesting fact about Sumudu transform is that the original function and its Sumudu transform have the same 
Taylor coefficients except a factor $n$ !. Thus if

$$
f(t)=\sum_{n=0}^{\infty} a_{n} t^{n}
$$

then

$$
F(u)=\sum_{n=0}^{\infty} n ! a_{n} t^{n}
$$

see (J. Zhang, 2007). Similarly, the Sumudu transform sends combinations, $C(m, n)$, into permutations, $P(m, n)$ and hence it will be useful in the discrete systems. Further

$$
S(H(t)=£(\delta(t))=1
$$

and

$$
£(H(t))=S(\delta(t))=\frac{1}{u} .
$$

Thus we further note that since many practical engineering problems involve mechanical or electrical systems acted upon by discontinuous or impulsive forcing terms then the Sumudu transform can be effectively used to solve ordinary differential equations as well as partial differential equations in engineering problems.

The Riemann-Liouville fractional integral of order $v$ is defined by Miller and Ross (K.S. Miller, 1993).

$$
{ }_{0} D_{t}^{-v} f(t)=\frac{1}{\Gamma(v)} \int_{0}^{t}(t-u)^{v-1} f(u) d u, \operatorname{Re}(v)>0 .
$$

Here we define the fractional partial-derivative for $\alpha>0$ in the form

$$
\frac{\partial^{\alpha} f(x, t)}{\partial t^{\alpha}}=\frac{1}{\Gamma(n-\alpha)} \frac{\partial^{n}}{\partial t^{n}} \int_{0}^{t} \frac{f(u, x) d u}{(t-u)^{\alpha-n+1}} ; n=[\alpha]+1
$$

where $[\alpha]$ means the integral part of the number $\alpha$. In particular, if $0 \leq \alpha<1$,

$$
\frac{\partial^{\alpha} f(t)}{\partial t^{\alpha}}=\frac{1}{\Gamma(1-\alpha)} \frac{\partial}{\partial t} \int_{0}^{t} \frac{f(u, x) d u}{(t-u)^{\alpha}}
$$

and if $\alpha=n, n \in N=\{1,2, \ldots\}$, then we have the ordinary derivative

$$
\frac{\partial^{n} f(t)}{\partial t^{n}}
$$

A generalization of the Mittag-Leffler function is given by

$$
\mathcal{E}_{\alpha}(z)=\sum_{n=0}^{\infty} \frac{z^{n}}{\Gamma(n \alpha+1)}, \quad \alpha \in C, \operatorname{Re}(\alpha)>0
$$

and the general form as follows

$$
\mathcal{E}_{\alpha, \beta}(z)=\sum_{n=0}^{\infty} \frac{z^{n}}{\Gamma(n \alpha+\beta)}, \quad \alpha, \beta \in C, \operatorname{Re}(\alpha)>0, \operatorname{Re}(\beta)>0 .
$$

In order to prove our main results, we shall require the following lemma that was proved in (V. G. Gupta, 2010).

Lemma 1. Sumudu transform of Mittag-leffer function $\mathcal{E}_{\alpha, \beta}(z)=\sum_{n=0}^{\infty} \frac{z^{n}}{\Gamma(n \alpha+\beta)}$, Re $\alpha>0$ and $\operatorname{Re} \beta>0$ exists and given by

$$
S\left[t^{\gamma-1} \mathcal{E}_{\beta, \gamma}\left(\omega t^{\beta}\right)\right]=u^{\gamma-1}\left(1-\omega u^{\beta}\right) .
$$

By using the above lemma we note that Mittag-leffer function can be recovered as follows: 
Lemma 2. Let $\mathcal{E}_{\alpha, \beta}(z)=\sum_{n=0}^{\infty} \frac{z^{n}}{\Gamma(n \alpha+\beta)}$ then

$$
S^{-1}\left[\frac{1}{u\left(u^{-\alpha}+a u^{-\beta}+b\right)}\right]=\frac{1}{\sqrt{a^{2}-4 b}} \tau^{\alpha-1}\left[\mathcal{E}_{\alpha, \alpha}\left(\lambda_{1} \tau^{\alpha}\right)-\mathcal{E}_{\alpha, \alpha}\left(\lambda_{2} \tau^{\alpha}\right)\right]
$$

where

$$
\lambda_{1}=\frac{1}{2}\left(-a+\sqrt{a^{2}-4 b}\right)
$$

and

$$
\lambda_{2}=\frac{1}{2}\left(-a-\sqrt{a^{2}-4 b}\right) .
$$

Proof. Since

$$
\begin{aligned}
\frac{1}{u\left(u^{-\alpha}+a u^{-\beta}+b\right)} & =\frac{1}{u\left(\lambda_{1}-\lambda_{2}\right)}\left(\frac{1}{u^{-\alpha}-\lambda_{1}}-\frac{1}{u^{-\alpha}-\lambda_{2}}\right) \\
& =\frac{1}{\left(\lambda_{1}-\lambda_{2}\right)}\left(\frac{u^{\alpha-1}}{1-\lambda_{1} u^{\alpha}}-\frac{u^{\alpha-1}}{1-\lambda_{2} u^{\alpha}}\right)
\end{aligned}
$$

where $\lambda_{1}$ and $\lambda_{2}$ are given by (10) and (11), respectively. Now taking inverse Sumudu transform and using Lemma 1, we get the desired result.

\section{Maxwell's equations of time-fractional order and Sumudu transform}

Maxewell's equations are fundamental equations in electricity and magnetism to find electromagnetic fields that can exist in any configuration (normal modes). Thus we can develop most of the working relationships in the field. Also they have successfully been applied to predict the electromagnetic fields that are produced by any distribution of charges and currents. The equations are general and no restriction is placed on the type of variation of the exciting sources. In the present paper, we introduce the Maxwell's equations of time-fractional order.

Now consider the transverse electromagnetic planar where the waves propagating in the z-direction having unbounded lossy medium is given by

$$
\begin{aligned}
\frac{\partial E_{x}}{\partial z}+\mu \frac{\partial H_{y}}{\partial t} & =0 \\
\frac{\partial H_{y}}{\partial z}+\epsilon \frac{\partial E_{x}}{\partial t}+\sigma E_{x} & =0
\end{aligned}
$$

where $\mu, \epsilon$ and $\sigma>0$ are the constants permeability, permittivity and the conductivity, respectively, see (M. E. ElShandwily, 1988). The boundary conditions are given as follows

$$
\begin{aligned}
E_{x}(\infty, t) & =\text { finite } \\
E_{x}(z, 0) & =H_{y}(z, 0)=0 \\
\frac{\partial E_{x}(z, 0)}{\partial z} & =\frac{\partial H_{y}(z, 0)}{\partial z}=0 \\
\left.\frac{\partial E_{x}(z, t)}{\partial t}\right|_{t=0} & =\left.\frac{\partial H_{y}(z, t)}{\partial t}\right|_{t=0}=0
\end{aligned}
$$

and

$$
E(0, t)=\left\{\begin{array}{cc}
0, & t<0 \\
f(t), & t \geq 0
\end{array} .\right.
$$

We note that many authors have discussed the solution of (12) and (13) by using different method, for example see (M. E. El-Shandwily, 1988) and (M. G. M. Hussain, 2007).

Now we consider the Maxwell's equations with time-fractional order under the same boundary conditions as follows

$$
\begin{aligned}
\frac{\partial E_{x}}{\partial z}+\mu \frac{\partial^{\alpha} H_{y}}{\partial t^{\alpha}} & =0 \\
\frac{\partial H_{y}}{\partial z}+\epsilon \frac{\partial^{\alpha} E_{x}}{\partial t^{\alpha}}+\sigma E_{x} & =0
\end{aligned}
$$


where $0 \leq \alpha<1$. Taking the Sumudu transform of the above equations (14) and (15), we get

$$
\begin{aligned}
\frac{d \bar{E}_{x}(z, u)}{d z}+\mu\left[\frac{\bar{H}_{y}(z, u)}{u^{\alpha}}-\frac{H(z, 0)}{u^{\alpha}}\right] & =0 \\
\frac{d \bar{H}_{y}(z, u)}{d z}+\epsilon\left[\frac{\bar{E}_{x}(z, u)}{u^{\alpha}}-\frac{E_{x}(z, 0)}{u^{\alpha}}\right]+\sigma \bar{E}_{x}(z, u) & =0 .
\end{aligned}
$$

Now by eliminating $\bar{H}(z, u)$ from (16) and (17), we get

$$
\frac{d^{2} \bar{E}_{x}(z, u)}{d z^{2}}-\left[\frac{\mu \epsilon}{u^{2 \alpha}}+\frac{\sigma \mu}{u^{\alpha}}\right] \bar{E}_{x}(z, u)=\frac{\mu}{u^{\alpha}} \frac{\partial H(z, 0)}{\partial z}-\frac{\mu \epsilon}{u^{2 \alpha}} E(z, 0) .
$$

By applying boundary conditions, then we obtain the equation in following form

$$
\frac{d^{2} \bar{E}_{x}(z, u)}{d z^{2}}-\left[\frac{\mu \epsilon}{u^{2 \alpha}}+\frac{\sigma \mu}{u^{\alpha}}\right] \bar{E}_{x}(z, u)=0 .
$$

By applying Sine transform to the above equation (19), we get

$$
\left(-s^{2} \bar{E}_{s}^{*}(s, u)+s E(0, u)\right)-\left(\frac{\mu \epsilon}{u^{2 \alpha}}+\frac{\sigma \mu}{u^{\alpha}}\right) \bar{E}_{s}^{*}(s, u)=0
$$

where $\bar{E}_{s}^{*}(s, u)=\sqrt{\frac{2}{\pi}} \int_{0}^{\infty} E(z, u) \sin s z d z$. This implies

$$
\bar{E}_{s}^{*}(s, u)=\frac{s f(u)}{s^{2}+\mu \epsilon u^{-2 \alpha}+\mu \sigma u^{-\alpha}} .
$$

Now by taking the inverse Sumudu transform and using convolution theorem, we obtain

$$
\bar{E}_{s}^{*}(s, u)=\frac{s}{\mu \epsilon \sqrt{a^{2}-4 b}} \int_{0}^{t} \tau^{\alpha-1}\left[\mathcal{E}_{\alpha, \alpha}\left(\lambda_{1} \tau^{\alpha}\right)-\mathcal{E}_{\alpha, \alpha}\left(\lambda_{2} \tau^{\alpha}\right)\right] f(\tau-t) d \tau
$$

where $\lambda_{1}$ and $\lambda_{2}$ are given in (9) and (10) and $a=\sigma_{\epsilon}$ and $b=s^{2} / \mu \epsilon$. Now taking the inverse sine transform we obtain

$$
E(z, t)=\int_{0}^{\infty} \frac{s}{\mu \epsilon \sqrt{a^{2}-4 b}}(\sin s z) \int_{0}^{t} \tau^{\alpha-1}\left[\mathcal{E}_{\alpha, \alpha}\left(\lambda_{1}, \tau^{\alpha}\right)-\mathcal{E}_{\alpha, \alpha}\left(\lambda_{2}, \tau^{\alpha}\right)\right] f(\tau-t) d \tau d s,
$$

which is the desired solution.

For magnetic field, by using the equation (16), and substituting the value of (22), we easily get

$$
\bar{H}(z, u)=-\frac{1}{\mu \epsilon} \int_{0}^{\infty} s^{2} \cos s z\left(\frac{u^{\alpha} f(u)}{u\left(u^{-2 \alpha}+a u^{-\alpha}+b\right)}\right) d s .
$$

Taking the inverse Sumudu transform and by using the Lemma 1, we obtain

$$
H(z, t)=\int_{0}^{\infty} \frac{s^{2}}{\mu \epsilon \sqrt{a^{2}-4 b}}(\cos s z) \int_{0}^{t} \tau^{2 \alpha-1}\left[\mathcal{E}_{\alpha, 2 \alpha}\left(\lambda_{1} \tau^{\alpha}\right)-\mathcal{E}_{\alpha, 2 \alpha}\left(\lambda_{2} \tau^{\alpha}\right)\right] f(\tau-t) d \tau d s .
$$

\section{Acknowledgment}

The first author acknowledge that this research was partially supported by the University Putra Malaysia under the Research University Grant Scheme(RUGS) 05-01-09-0720RU.

\section{References}

A. Kadem. (2005). Solving the one-dimensional neutron transport equation using Chebyshev polynomials and the Sumudu transform. Analele Universitatii din Oradea. Fascicola Matematica, Vol. XII, pp. 153-171. 
A. Kılıçman and H. Eltayeb. (2010). A note On Integral Transforms and Partial Differential Equations. Applied Mathematical Sciences, 4(3), pp. 109-118.

A. Kılıçman and H. Eltayeb. (2010). On the applications of Laplace and Sumudu transforms. Journal of the Franklin Institute, vol. 347, no. 5, pp. 848-862. doi:10.1016/j.jfranklin.2010.03.008.

A. Kılıçman, H. Eltayeb and P. Ravi Agarwal. (2010). On Sumudu Transform and System of Differential Equations. Abstract and Applied Analysis, Volume 2010, Article ID 598702, 11 pages doi:10.1155/2010/598702.

F. B. M. Belgacem, A. A. Karaballi, and S. L. Kalla. (2003). Analytical investigations of the Sumudu transform and applications to integral production equations. Math. Prob. Eng., (3), pp. 103-118.

G. Jumarie. (2007). Fractional partial differential equations and modified RiemannLiouville derivatives. Method for solution. J. Appl. Math. Computing, 24 (12), pp. 31-48.

G. Jumarie. (2009). Table of some basic fractional calculus formulae derived from a modified RiemannLiouville derivative for non-differentiable functions. Appl. Math. Lett., 22(3), pp. 378-385.

G. Jumarie. (2009). Laplace transform of fractional order via the MittagLeffler function and modified RiemannLiouville derivative, Appl. Math. Lett., 22(11), pp. 1659-1664.

G. K. Watugala. (1993). Sumudu transform: a new integral transform to solve differential equations and control engineering problems, Int. J. Math. Edu. Sci. Tech., 24 (1), pp. 35-43.

H. Eltayeb, A. Kılıçman, and B. Fisher. (2010). A new integral transform and associated distributions, Int. Trans. Spec. Func., 21(5), pp. 367-379.

J. M. Tchuenche and N. S. Mbare. (2007). An application of the double Sumudu transform. Applied Mathematical Sciences, 1(1), pp. 31-39.

J. Zhang. (2007). A Sumudu based algorithm for solving differential equations. Computer Science Journal of Moldova, 15(3), pp. 303-313.

K.S. Miller and B. Ross. (1993). An Introduction to the Fractional Calculus and Fractional Differential Equations, John Wiley and Sons, New York.

M. A. Asiru. (2001). Sumudu transform and the solution of integral equations of convolution type. Int. J. Math. Edu. Sci. Tech., 32(6), pp. 906-910.

M. A. Asiru. (2002). Further properties of the Sumudu transform and its applications. Int. J. Math. Edu. Sci. Tech., 33(3), pp. 441-449.

M. A. Asiru. (2003). Classroom note: application of the Sumudu transform to discrete dynamic systems. Int. J. Math. Edu. Sci. Tech., 34(6), pp. 944-949.

M. Caputo. (1969). Elasticita e Dissipazione, Zanichelli, Bologna.

M. E. El-Shandwily. (1988). Solutions of Maxwells equations for general nonperiodic waves in lossy media. IEEE Trans. Electromagn. Compat., 30(4), pp. 577-582.

M. G. M. Hussain and F. B. M. Belgacem. (2007). Transient solutions of Maxwell's equations based on Sumudu transform. Progress In Electromagnetics Research(PIER), (74), pp. 273-289.

V. G. Gupta and B. Sharma. (2010). Application of Sumudu Transform in Reaction-Diffusion Systems and Nonlinear Waves. Appl. Math. Sci., 4(9), pp. 435-446. 